\title{
ANALYSIS OF MHD NON-DARCIAN BOUNDARY LAYER FLOW AND HEAT TRANSFER OVER AN EXPONENTIALLY VERTICALLY STRTCHING SURFACE WITH THERMAL RADIATION
}

\author{
M. Subhas Abel ${ }^{1}$, Veena.M.Basangouda ${ }^{2}$, M.Narayana ${ }^{3}$, Prashant.G.metri ${ }^{4}$ \\ ${ }^{1}$ Dept of Mathematics Gulbarga University, Gulbarga 585106, Karnataka, India \\ ${ }^{2}$ Dept of Mathematics Gulbarga University, Gulbarga 585106, Karnataka, India \\ ${ }^{3}$ School of Advanced science, VIT University, Vellore 632014, Tamilnadu, India \\ ${ }^{4}$ Dept of Mathematics Gulbarga University, Gulbarga 585106, Karnataka, India
}

\begin{abstract}
This paper deals with the numerical study of MHD Non-Darcianlayer flow on an exponentially stretching surface and free convection heat transfer with a presence of Thermal Radiation. The flow is considered over a stretching sheet in the presence of non dimensional parameters. Conversion of governing nonlinear boundary layer equations to coupled higher order non-linear ordinary differential equations using similarity transformations. The obtained governing equations were solved numerically by using keller box method. The various nondimentional parameters effects with velocity profile and thermal profile are discussed in detail with graphically.
\end{abstract}

Keywords: Thermal Radiation, Statching Surface

\section{INTRODUCTION}

In industrial manufacturing process the heat and mass transfer problems are well used. This phenomena applicable in wire and fibre coatings and transpiration cooling etc. In astrophysics and geophysics the MHD flow basically used. Basically the MHD flow has wide applications. Usually used in Engineering and industrial.T he fluid subjected to a magnetic field become a good agreement results. There is a wide application in Mechanical Engineering field.. After the pioneering work of Sakiadis $[1,2]$ many researchers gave attention to study flow and heat transfer of Newtonian and non-Newtonian fluids over a linear stretching sheet. By considering quadratic stretching sheet, Kumaran and Ramanaiah [3] analyzed the problem of heat transfer. Ali [4] investigated the thermal boundary layer flow on a power law stretching surface with suction or injection.

Elbashbeshy [5] analyzed the problem of heat transfer over an exponentially stretching sheet with suction. Magyari and Keller [6] discussed the heat and mass transfer in boundary layers on an exponentially stretching continuous surface. Sanjayanand and Khan [7, 8] extended the work of Elbashbeshy [5] to viscoelastic fluid flow, heat and mass transfer over an exponentially stretching sheet .Raptis et al.[9] constructed similarity solutions for boundary layer near a vertical surface in a porous medium with constant temperature and concentration. Bejan and Khair [10] used Darcy's law to study the features of natural convection boundary layer flow driven by temperature and concentration gradients. Forchheimer[11] proposed quadratic term in Darcian velocity to describe the inertia effect in porous medium. Plumb and Huenefeld[12] studied the problem of non-Darcian free convection over a vertical isothermal flat plate. Rees and Pop[13] also studied yhe free convection flow along a vertical wavy surface with constant wall temperature. Rees and Pop[14] studied the case where the heated surface displays waves while the Darcys law is supplemented by the Forchheimerterms. They stated that the boundary flow remains self similar in the presence of surface waves but where inertia is absent, and when inertia is present but surface waves are absent. However, the combination of the two effects yields non similarity. Tsou et al.[15] studied flow and heat transfer in the boundary layer on a continuous moving surface while Gupta and Gupta[16] solved boundary layer flow with suction and injection. Andresson and Bech[17] have studied the MHD flow of the power law fluid over stretching sheet. Pavlov[18] gave an exact similarity solution to the MHD boundary layer equation for the steady and two dimensional flow caused solely by the stretching if an elastic surface in the presence of uniform magnetic field. M S Abel and Mahesha [19] heat transfer in MHD visco elastic fluid flow over a stretching sheet with variable thermal conductivity non uniform heat, source andradiation. In the paper we analysed thermal radiation effect in a exponentially vertically stretching surface on a MHD flow. And effect of various physically parameters are also discussed in detail. 


\section{MATHEMATICAL FORMULATION}

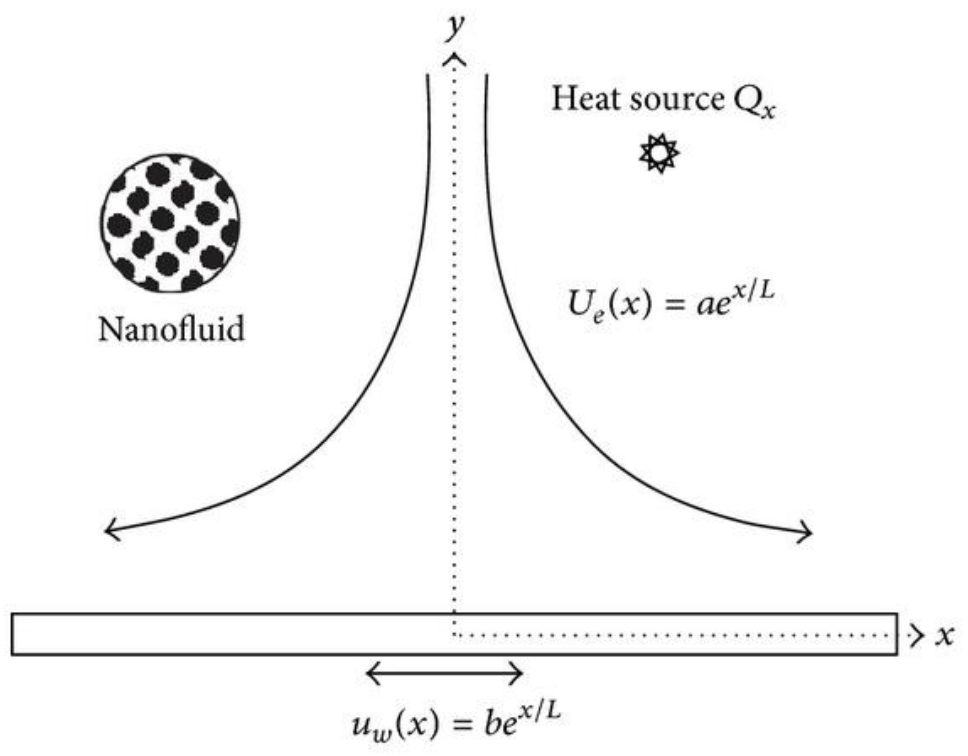

Under the usual boundary layer approximations, the flow and heat transfer in the presence of radiation effects are governed by the following equations:

$$
\begin{gathered}
\frac{\partial u}{\partial x}+\frac{\partial v}{\partial y}=0 \\
u \frac{\partial u}{\partial x}+v \frac{\partial u}{\partial y}=v \frac{\partial^{2} u}{\partial y^{2}}-\frac{v}{k} u-\frac{C_{b}}{\sqrt{k}} u^{2}+g \beta\left(T-T_{\infty}\right)-\sigma \frac{B_{0}^{2}}{\rho} u \\
u \frac{\partial T}{\partial x}+v \frac{\partial T}{\partial y}=\alpha \frac{\partial^{2} T}{\partial y^{2}}+\frac{\sigma}{\rho C_{p}} B_{0}^{2} u^{2}+\frac{\mu}{\rho C_{p}}\left(\frac{\partial u}{\partial y}\right)^{2}+\rho \frac{Q}{\rho C_{p}}\left(T-T_{\infty}\right)-\frac{1}{\rho c_{p}} \frac{\partial q_{r}}{\partial y}
\end{gathered}
$$

The associated boundary conditions to the problem are

$$
\begin{gathered}
\mathrm{U}=U_{w}(x), v=0, T=T_{w}(x), \text { at } \mathrm{y}=0, \\
u=0, T \rightarrow T_{\infty} \text { as } y \rightarrow \infty \\
U_{w}(x)=U_{0} e^{\frac{x}{L}}, \\
T_{w}(x)=T_{\infty}+\left(T_{0}-T_{\infty}\right) e^{\frac{a x}{2 L}},
\end{gathered}
$$


Where $T_{0}$ and a are parameters of temperature distribution in the stretching surface. $\mathrm{T}$ is the temperature, $\mathrm{K}$ is the thermal conductivity, $C_{p}$ is the Specific heat and $q_{r}$ is the radiative heat flux.

$$
q_{r}=-\frac{4 \sigma *}{3 K^{*}} \frac{\partial T^{4}}{\partial y}
$$

Where $K^{*}$ is the mean absorption coefficient and $\sigma *$ is the Stefan-Boltzmann Constant. $T^{4}$ is expressed as a linear function of temperature, hence

$$
T^{4}=4 T_{\infty}^{3} T-3 T_{\infty}^{4}
$$

Introducing the following non- dimensional parameter

$$
\begin{gathered}
\eta=\sqrt{\frac{\operatorname{Re} y}{2 L}} e^{\frac{x}{2 L}}, \psi(x, \eta)=\sqrt{2 \operatorname{Re} v} e^{\frac{x}{2 L}} f(\eta), \\
T(x, y)=T_{\infty}+\left(T_{0}-T_{\infty}\right) e^{\frac{a x}{2 L}} \theta(\eta),
\end{gathered}
$$

Where $\psi$ is the stream function which is defined in the usual form as

$$
u=\frac{\partial \psi}{\partial y}_{\&} v=-\frac{\partial \psi}{\partial x}
$$

Substituting (10)\&(11) in (12).We obtain $\mathrm{u}$ and $\mathrm{v}$ as follows

$u(x, y)=u_{0} e^{\frac{x}{L}} f^{\prime}(\eta), v(x, y)=-\frac{v}{L} \sqrt{\frac{\operatorname{Re}}{2}} e^{\frac{x}{2 L}}\left[f(\eta)+\eta f^{\prime}(\eta)\right]$

Eqns (1) to(5) istronsformed into the ordinary differential equation with the aid of equations( 10)-(13).Thus, the governing equations using the diemensionalessfuction $\mathrm{f}(\eta)$ and $\theta(\eta)$ become 


$$
f^{\prime \prime \prime}+f f^{\prime \prime}-\left(2+N_{2}\right) f^{\prime 2}+2 G r e^{\frac{a x}{2}} e^{-2 x} \theta-2 e^{-x} f^{\prime}\left(\frac{H a^{2}}{\operatorname{Re}}+N_{1}\right)=0
$$

$\operatorname{Pr}^{-1} \theta^{\prime \prime}\left(1+\frac{4 K}{3}\right)+f \theta^{\prime}-a f^{\prime} \theta+e^{\frac{X(2-a)}{2}} E c\left(2 \frac{H a^{2}}{\operatorname{Re}} f^{\prime 2}+f^{\prime \prime 2} e^{X}\right)+2 \lambda e^{-X} \theta=0$

The boundary conditions (4) and (5) reduce to

$$
\begin{gathered}
C_{f} \sqrt{\operatorname{Re}_{x}}=\sqrt{2 X} f^{\prime \prime}(0) . \mathrm{f}(0)=0, \mathrm{f}^{\prime}(0)=1, \theta(0)=1, \\
f^{\prime}(\infty)=0, \theta(\infty)=0
\end{gathered}
$$

Where, $\quad X=\frac{x}{L} H a=\left(\frac{\sigma B_{0}^{2} L^{2}}{\rho v}\right)^{\frac{1}{2}} \quad$ is $\quad$ Hartman $\quad$ number, $E c=U_{0}^{2} / c_{p}\left(T_{0}-T_{\infty}\right) \quad$ is $\quad$ Eckert

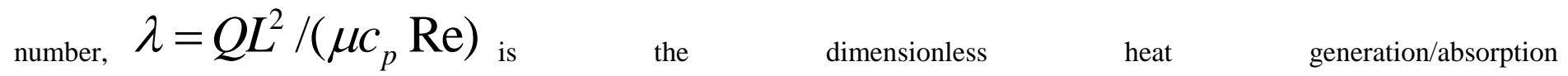
parameter, $G r_{1}=g \beta\left(T_{0}-T_{\infty}\right) \frac{L^{3}}{v^{2}} \quad$ is $\quad$ the $\quad$ Grashof $\quad$ number, $\operatorname{Re}=U_{0} L / v \quad$ is $\quad$ Reynolds number, $\mathrm{Gr}=\mathrm{Gr}_{1} / \mathrm{Re}^{2}$ is the thermal buoyancy parameter, and $\operatorname{Pr}=\frac{\boldsymbol{\nu}}{\alpha} \quad$ is the Prandtlnumber,Where $N_{1}=\frac{L^{2}}{k \mathrm{Re}}$, is the porous parameter $N_{2}=\frac{2 C_{b} L}{\sqrt{k}}$ is the inertia coefficient $\lambda=L, Z=H a^{2} / \mathrm{Re}, a=W_{. \mathrm{K}=}$ $4 \sigma^{*} T_{\infty}^{3}$

$K^{*} K$ Radiation number.In the above system of local similarity equations , the effect of the magnetic field is included as a ratio of the Hartman number to the Reynolds number.

The physical quantities of interest in the problem are the local skin friction acting on the surface in contact with the ambient fluid of constant density which is defined as 


$$
\tau_{w x}=\rho v\left(\frac{\partial u}{\partial y}\right)_{y=0}=\left(\frac{\rho v U_{0}}{L}\right)\left(\frac{\operatorname{Re}}{2}\right)^{\frac{1}{2}} e^{\frac{x}{2}} f^{\prime \prime}(0)
$$

And the non-dimensional skin friction coefficient, $C_{f}$, which can be written as,

$$
C_{f}=\frac{2 \tau_{w x}}{\left(\rho U_{w}^{2}\right)} \text { or } C_{f} \sqrt{\mathrm{Re}_{x}}=\sqrt{2 X} f^{\prime \prime}(\mathrm{O})
$$

The local surface heat flux through the wall with $\mathrm{k}$ as thermal conductivity of the fluid is given by

$$
q_{w x}=-k\left(\frac{\partial T}{\partial y}\right)_{y=0}=\frac{k\left(T_{0}-T_{\infty}\right)}{L}\left(\frac{\operatorname{Re}}{2}\right)^{\frac{1}{2}} e^{\frac{(a+1)}{2}} \theta^{\prime}(0) .
$$

The local Nusselt number, $N u_{x}$, which is defined as

$$
\begin{gathered}
N u_{x}=\frac{x q_{w x}(x)}{k\left(T_{w}-T_{\infty}\right)}, \\
N u_{x} / \sqrt{\operatorname{Re}_{x}}=-(X / 2)^{\frac{1}{2}} \theta^{\prime}(0),
\end{gathered}
$$

Where $\operatorname{Re}_{x}$ is the local Reynolds number based on the surface velocity and is given by

$$
\operatorname{Re}_{x}=\frac{x U_{w}(x)}{v}
$$

\section{NUMERICAL METHOD}

The above Non linear equations that is 14 and 15 are subjected with similarity transformations and the obtained governing equations solved by finite difference scheme kellor box method by gauss elimination method.

$$
\begin{aligned}
& f^{\prime \prime \prime}+f f^{\prime \prime}-\left(2+N_{2}\right) f^{\prime 2}+2 G r e^{\frac{a x}{2}} e^{-2 x} \theta-2 e^{-x} f^{\prime}\left(\frac{H a^{2}}{\operatorname{Re}}+N_{1}\right)=0 \\
& \operatorname{Pr}^{-1} \theta^{\prime \prime}\left(1+\frac{4 K}{3}\right)+f \theta^{\prime}-a f^{\prime} \theta+e^{\frac{X(2-a)}{2}} E c\left(2 \frac{H a^{2}}{\operatorname{Re}} f^{\prime 2}+f^{\prime \prime 2} e^{X}\right)+2 \lambda e^{-X} \theta=0
\end{aligned}
$$




$$
\begin{aligned}
& f(0)=0, f^{\prime}(0)=1, \theta(0)=1 \text { as } \eta \rightarrow 0 \\
& f^{\prime}(\infty)=0, \theta(\infty)=0 \text { as } \eta \rightarrow \infty
\end{aligned}
$$

In this method the third and second order non linear differential equations,

\subsection{Finite Difference Scheme}

This scheme involves 5 steps

Step 1: Decomposing of given differential equations into a set of first order ordinary differential equations.

Step2: a) Approximate the first order derivatives with standard

forward difference $\frac{d y}{d x} \approx \frac{y_{i}-y_{i-1}}{\Delta x}$

b) Approximate the dependent variables with two point averages $y \approx \frac{y_{i}+y_{i-1}}{2}$ using these approximation the ordinary differential equations is transformed to finite difference equations. solution, say $y_{i}=\overline{y_{i}}+\delta y_{i}$ And substituting this in the finite difference equation and drop terms non-linear in $\delta y_{i}$ to arrive at linear F.D.E's.

Step3: Linearise F.D.E using Newton's method this involves to start with a guess seidel, or Jacobi method]and obtain $\delta y_{i}$ and add the correction to initial solution.

Step4: Solve the linearised F.D.E's using the standard method Gauss elimination.

Step 5.Repeat step 3 \& Step 4 until we obtain the required result.

\section{RESULTS AND DISCUSSIONS:}

Present results, are displayed in Table 1 and are noticed to be well in agreement with the present work

Fig. 2 Represents the effect of magnetic field parameter $\frac{H a^{2}}{\mathrm{Re}}$, on velocity profile $f^{\prime}$.Here magnetic field produces a drag in the form of Lorentz force.Due to this effect,the magnitude of velocity decreases and the thermal boundary layer thickness increases.

Fig 3 Represents the various values of parameter a with velocity profile. From this figure, it is observed that the value of a increases with increase in the velocity flow.and maximum velocity occurs at $\mathrm{a}=7$.
Fig. 4Represents the dimensionless parameter $\mathrm{X}$ with horizontal velocity profile. From this figure, it is noticed that the value of $\mathrm{X}$ increases with decreases in the velocity profile.here the flow is adjacent to a stretching sheet.

Fig. 5 It is observed from this figure that temperature decreases with increase in the values of a. Further, it is noticed that the thermal boundary layer thickness increases with increase in the value of a.. for positive value of a,heat transfer decreases. which indicates that, the flow of heat transfer is directed from the wall to the ambient fluid whereas the rate of heat transfer in the boundary layer increases near the wall.

Fig. 6 depicts the temperature profile in the fluid for various values of $\frac{H a^{2}}{\operatorname{Re}}$,for $\mathrm{a}=-2$ and $\mathrm{Gr}=0,0.5$. It is noticed that an increase in the strength of magnetic field i.e Lorentz force leads to an increase in the temperature far away from the wall, within the thermal boundary layer but the effect of magnetic field near the wall is to decrease the temperature in the absence of Grash of Number. When the magnetic field increases, the thermal boundary layer thickness increases.

fig7, and it is noticed that increase in Grash of number ,increase in temperature up to certain value of $\mathrm{n}$ and suddenly decreases and decays asymptotically to zero. Further it is observed that this increase in temperature is due to the temperature difference between stretched wall and the surrounding fluid. When Grash of number leads to increases, the thermal boundary layer thickness decreases

Fig. 8 Represents the temperature profile $\theta(\eta)$ for various values of $\mathrm{X}$ along $\eta$ for different values of a $=-1,-2$ and also Grash off number $\mathrm{Gr}=1.0$. It is noticed that the effect of increasing $\mathrm{X}$ on $\theta(\eta)$ is more effective for $\mathrm{a}=-2$ than compared to the results obtained in the case when a $=-1$. It is interesting to note the behaviour of $\mathrm{X}$ on $\theta(\eta)$, is that the temperature overshoots near the wall for small value of X,for a $=-2$, whereas the overshoot diminishes when a is enhanced to 1 for all other values of X. It is also observed that the boundary layer thickness decreases with an increase in X.

Fig. 9 Represents the variation of temperature profiles $\theta(\eta)$ for various values of magnetic field parameter $\left(\mathrm{Ha}^{2} / \mathrm{Re}=0,6\right.$, 8) for two values of $X$. when $X$ increases temperature decreases all other fixed values of other involved parameters 
except when the value of parameter $a=5$. It is also to be noticed that thermal boundary layer thickness increases as $\mathrm{X}$ decreases and the effect of magnetic field is to increase the temperature for both valuesof $\mathrm{X}$. This is due to the Lorentz force the temperature increases.

Fig10 Represents the effect of Prandtl number $\operatorname{Pr}$ on dimensionless heat transfer parameter $\theta$. It is noticed from this figure that as Prandtl number Pr increases, temperature profile decreases. When Prandtl number Pr is small, heat diffuses quickly compared to the velocity (momentum), especially for liquid metals,(low Prandtl number) the thickness of the thermal boundary layer is much bigger than the momentum boundary layer. Fluids with lower Prandtl number have higher thermal conductivities where.Hence the rate of cooling in conducting flows increases due to the Prandtl number.

Fig 11 Represents the effect of porous parameter N1 over velocity profile.Porous parameter increases, velocity decreases.Due to this, the velocity decreases in the boundary layer.
Fig12 Represents the effect of inertia coefficient N2 in the velocity profile.From this we conclude that due to the $\mathrm{N} 2$, the thickness of momentum of boundary layer decreases.

Fig 13: Represents the effect of heat source/sink parameter $\lambda$ .It is noticed that, when $\lambda>0$, the temperature increases. when, th $\lambda<0$ temperature falls.

Fig14: depicts dimensionless temperature field for various values of $\mathrm{K}$, with fixed values of other involved parameters. It is observed from the figure that, $\mathrm{K}$ increases, the temperature profiles and the thermal boundary layer thickness also increase.

Fig15: Effect of porous parameter N1 on a temperature profiles and it is noticed that, temperature increases with the increase of porous parameter, which offers resistance to the flow resulting in the increase of temperature in the boundary layer.

Fig16: Effect of drag coefficient of porous medium N2.From the figure it is noticed that the effect of drag coefficient is to increase the temperature profile in the boundary layer. Which implies boundary layer thickness also increases.

Table: Values of heat transfer coefficient, $\theta^{\prime}(\mathrm{O})$ for various values of $\mathrm{K}$ and Ec with $\operatorname{Pr}=1.0$ and all parameters taken as 0.0

\begin{tabular}{|l|l|l|c|}
\hline $\mathrm{K}$ & $\mathrm{Ec}=0.0$ & $\mathrm{Ec}=0.5$ & $\mathrm{Ec}=1.0$ \\
\hline 1.0 & -1.641723 & -0.6609 & 0.3198 \\
\hline 2.0 & -0.57579 & -0.29001 & -0.00423 \\
\hline 3.0 & -0.4714 & -0.26390 & -0.05638 \\
\hline
\end{tabular}




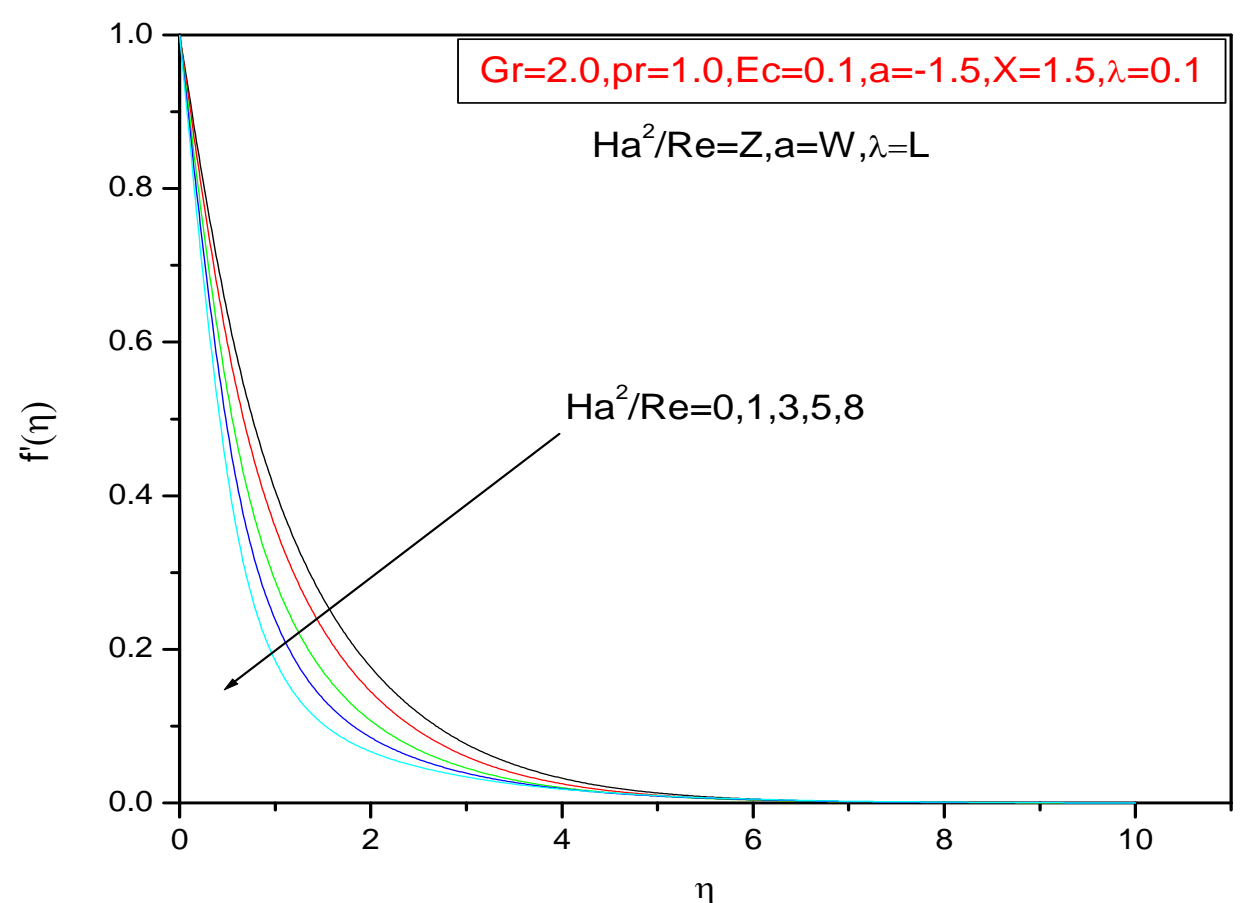

Fig 2 Effect of magnetic field on velocity profiles with $\eta$

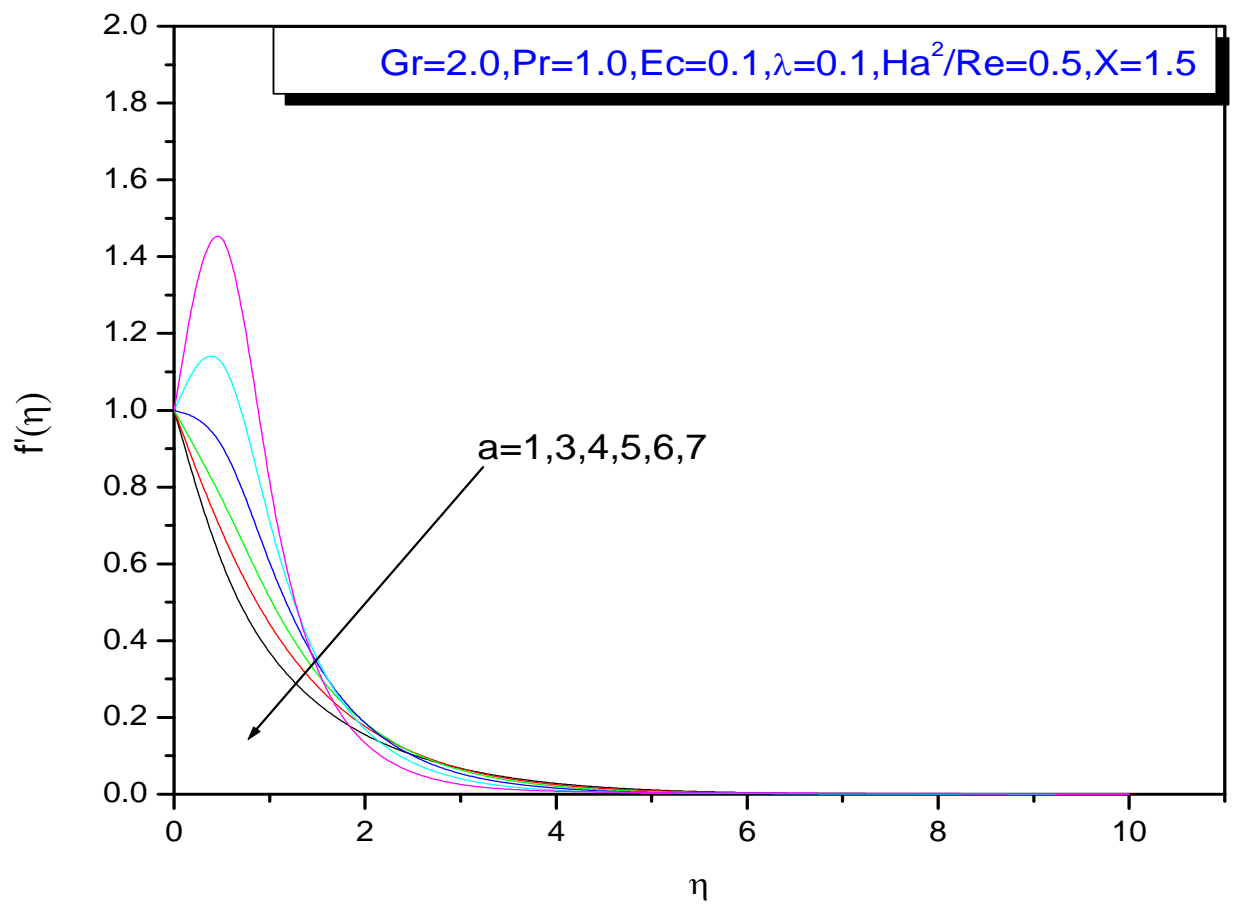

Fig.3. Variation of velocity profiles with $\eta$ for various values of a. 


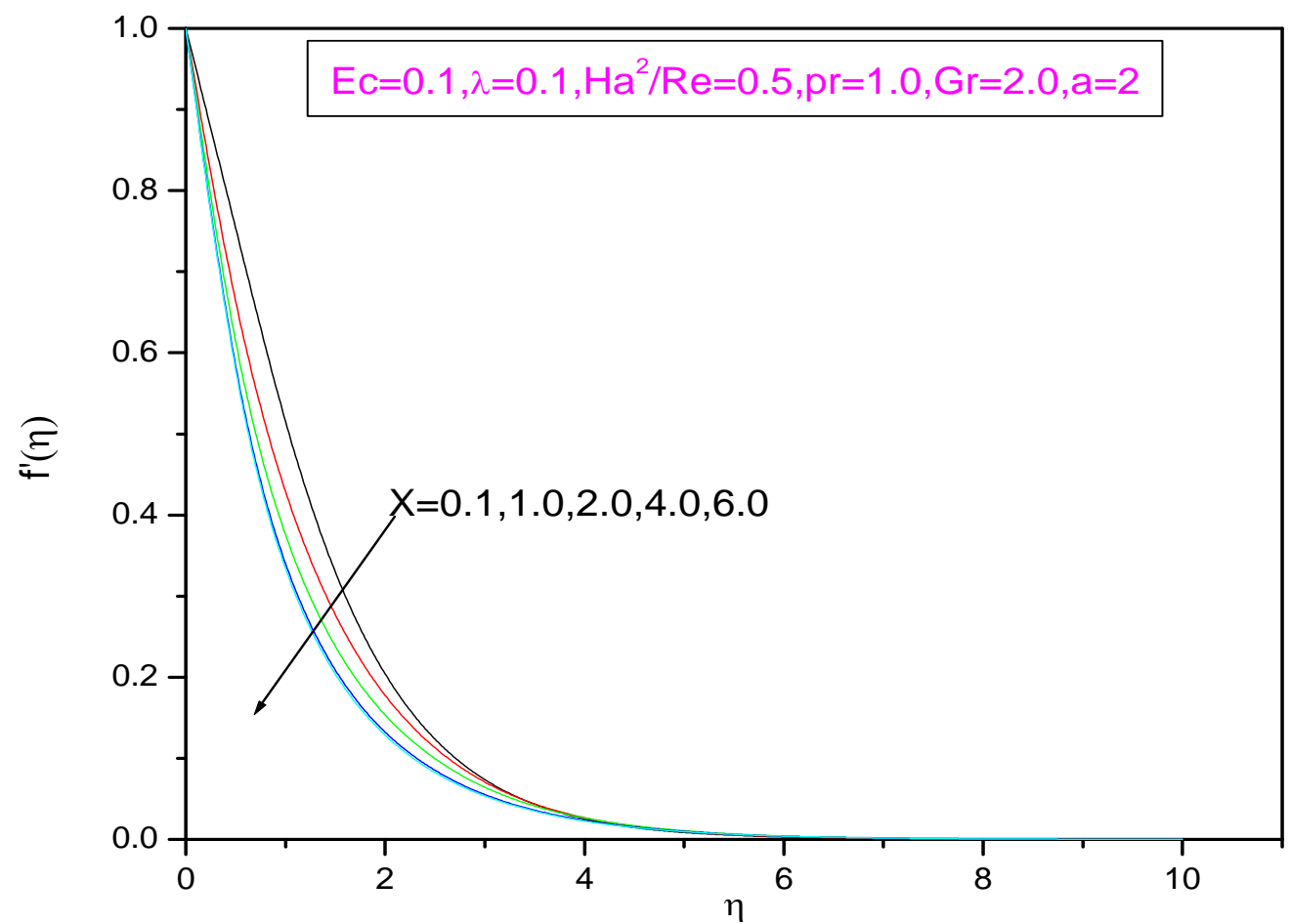

Fig.4.Variations of velocity profiles with $\eta$ for different values of $X$.

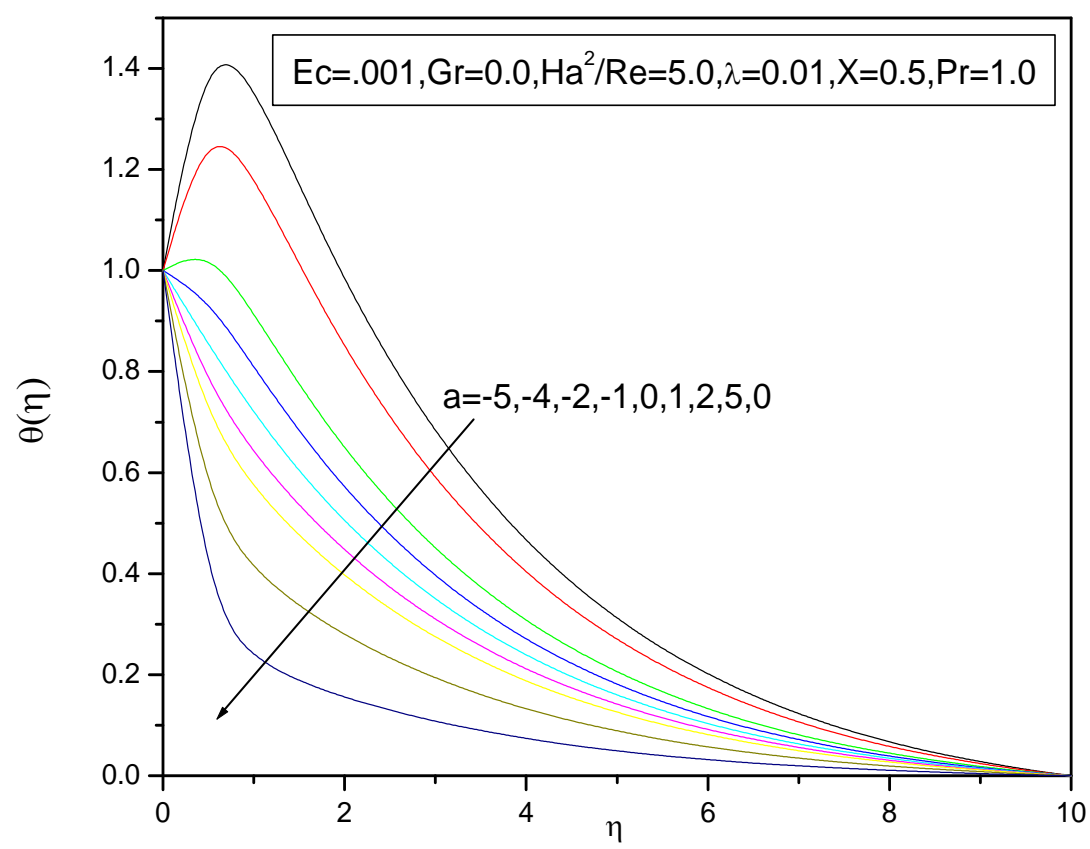

Fig.5.Temperature profiles vs. $\eta$ for various values of $a$. 


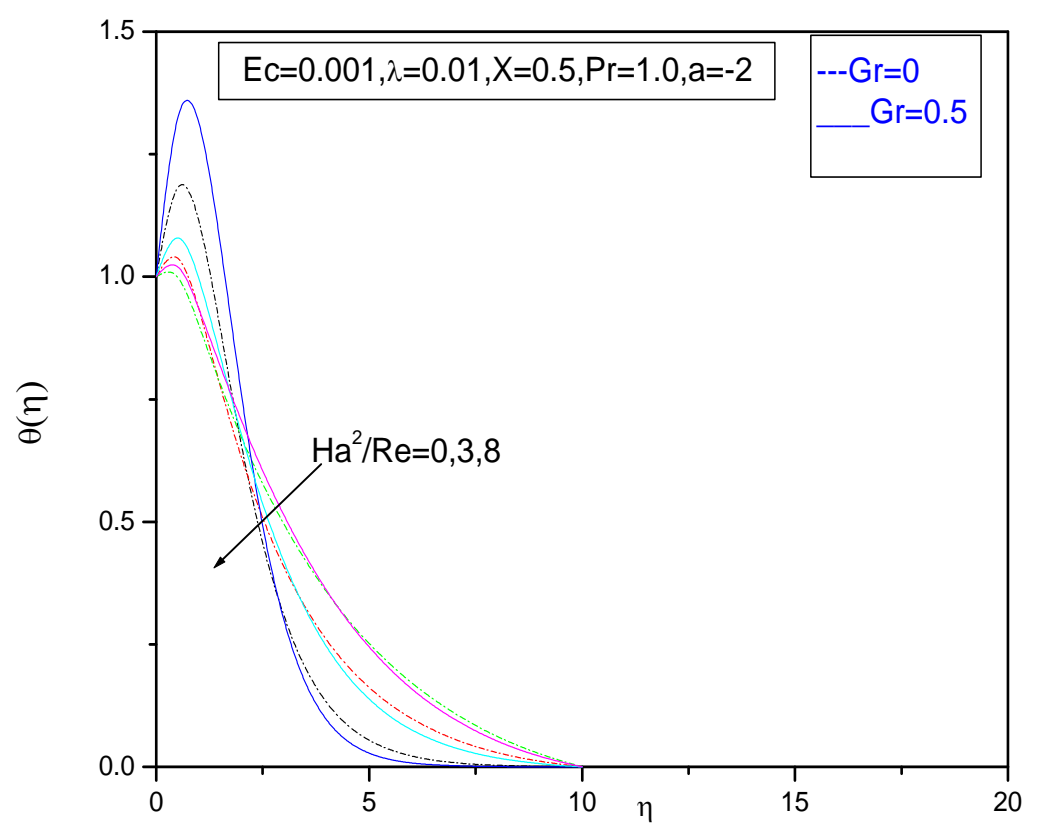

Fig.6.Temperature profiles vs. $\eta$ for various values of $\mathrm{Ha}^{2} / \mathrm{Re}$ and $\mathrm{Gr}$.

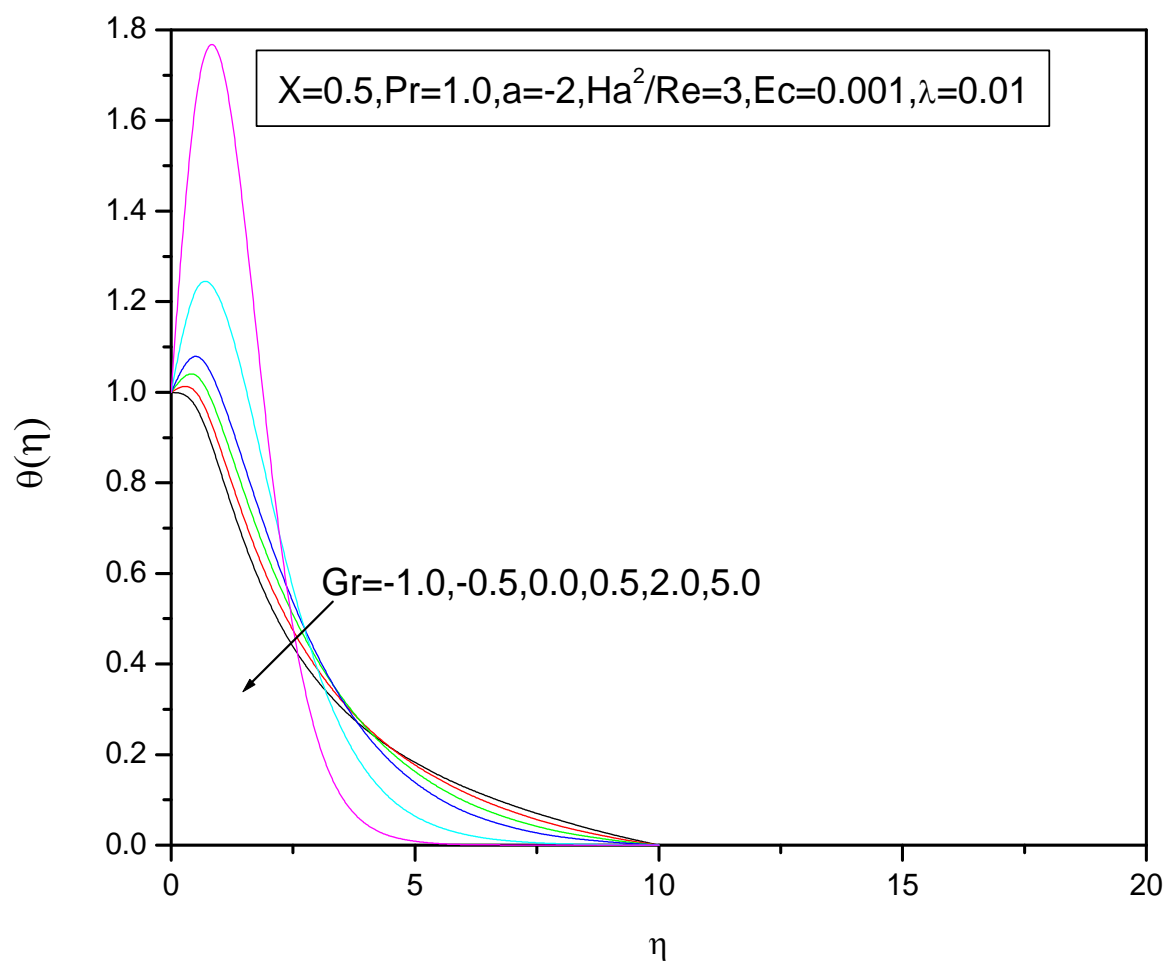

Fig7 temperature profile for various values of $\mathrm{Gr}$ 


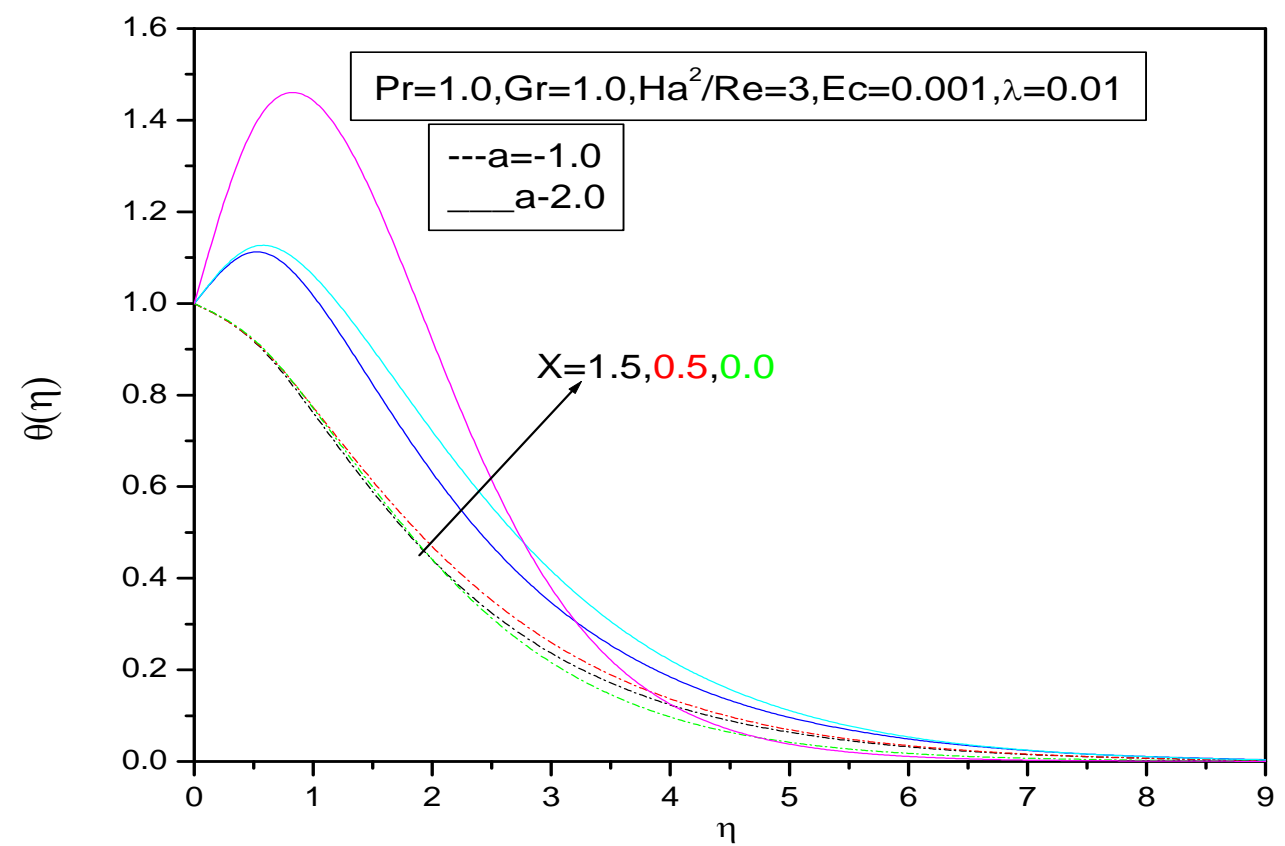

Fig.8.Temperature profiles vs. $\eta$ for various values of $a$ and $X$.

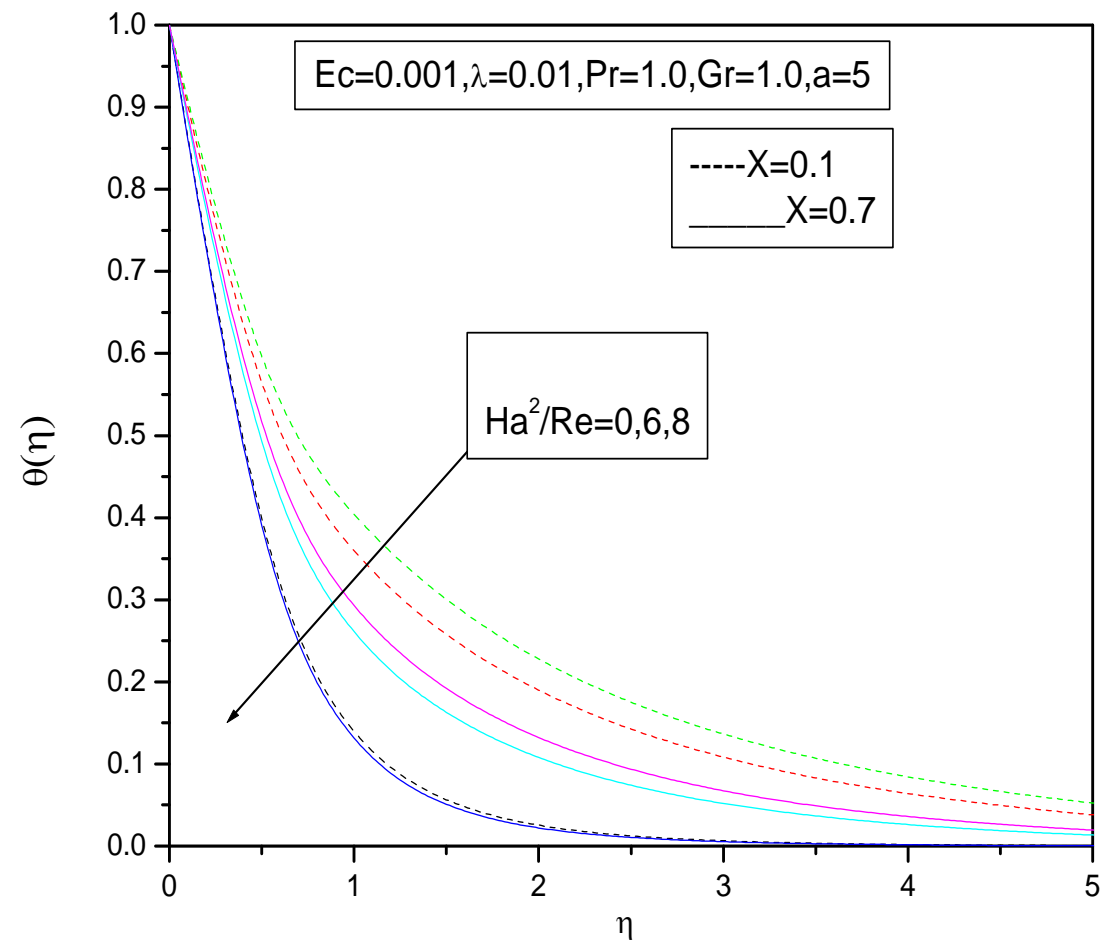

Fig.9.Temperature profiles vs. $\eta$ for various values of $\mathrm{Ha}^{2} / \mathrm{Re}$ and $\mathrm{X}$ when $\mathrm{a}=5$. 


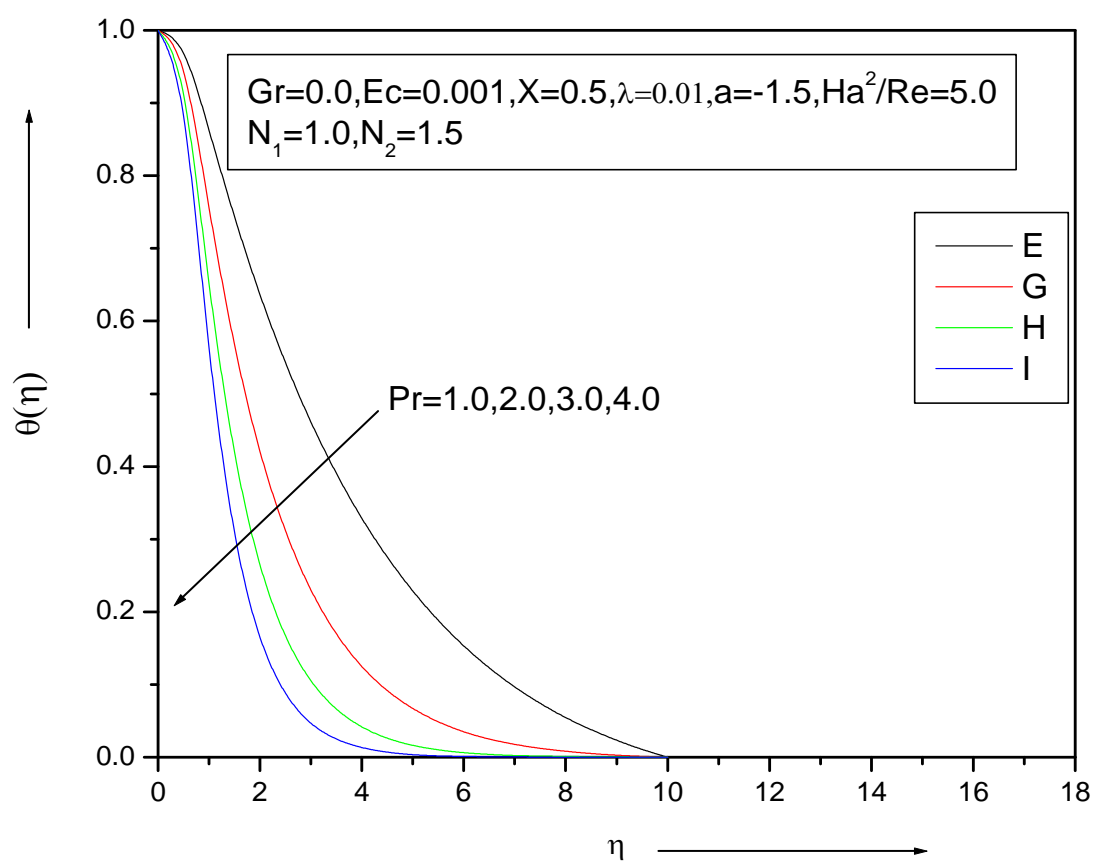

Fig10.Variation of temperature with $\eta$ for different values of $\operatorname{Pr}$

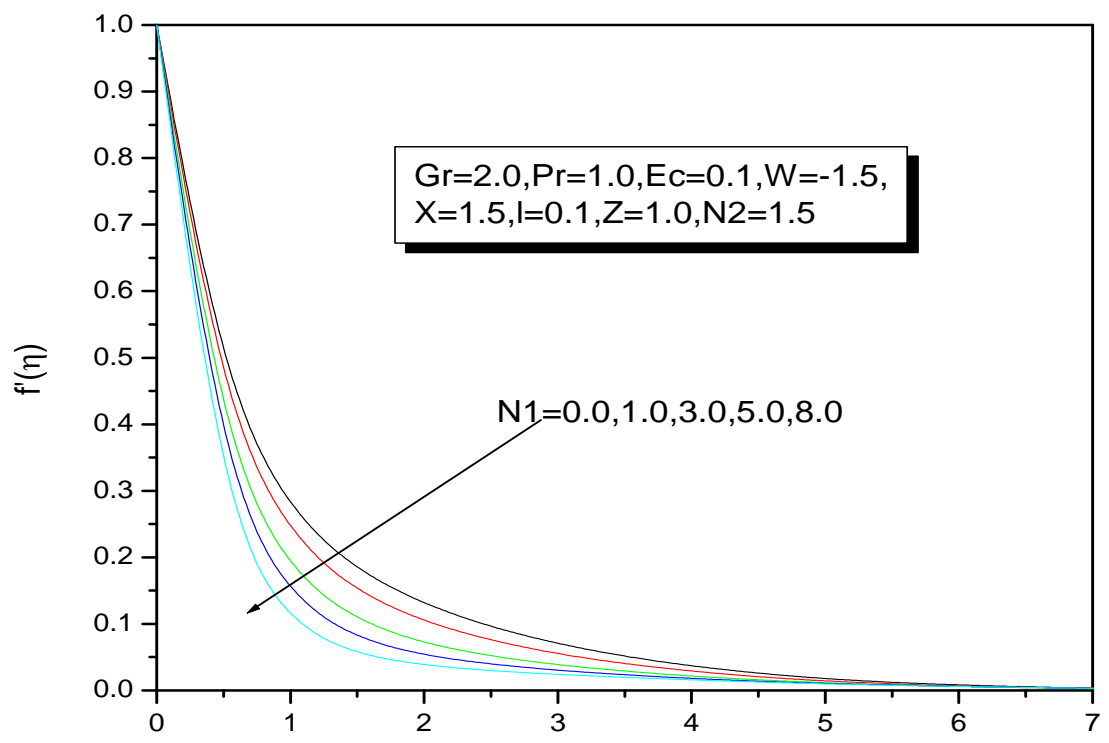

Fig.11. Temperature profile vs. $\eta$ for various values of $\mathrm{N} 1$. 


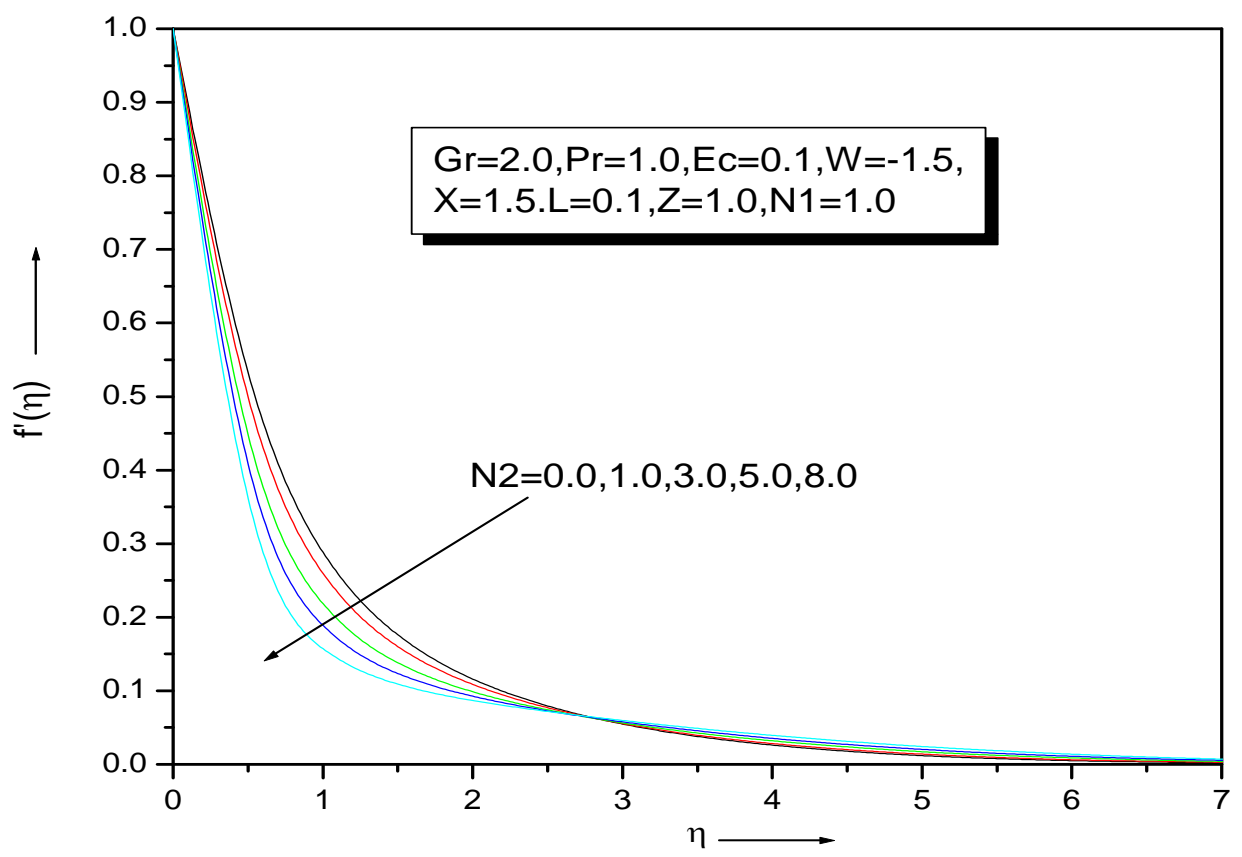

Fig.12. Temperature profile vs. $\eta$ various values of $\mathrm{N} 2$.

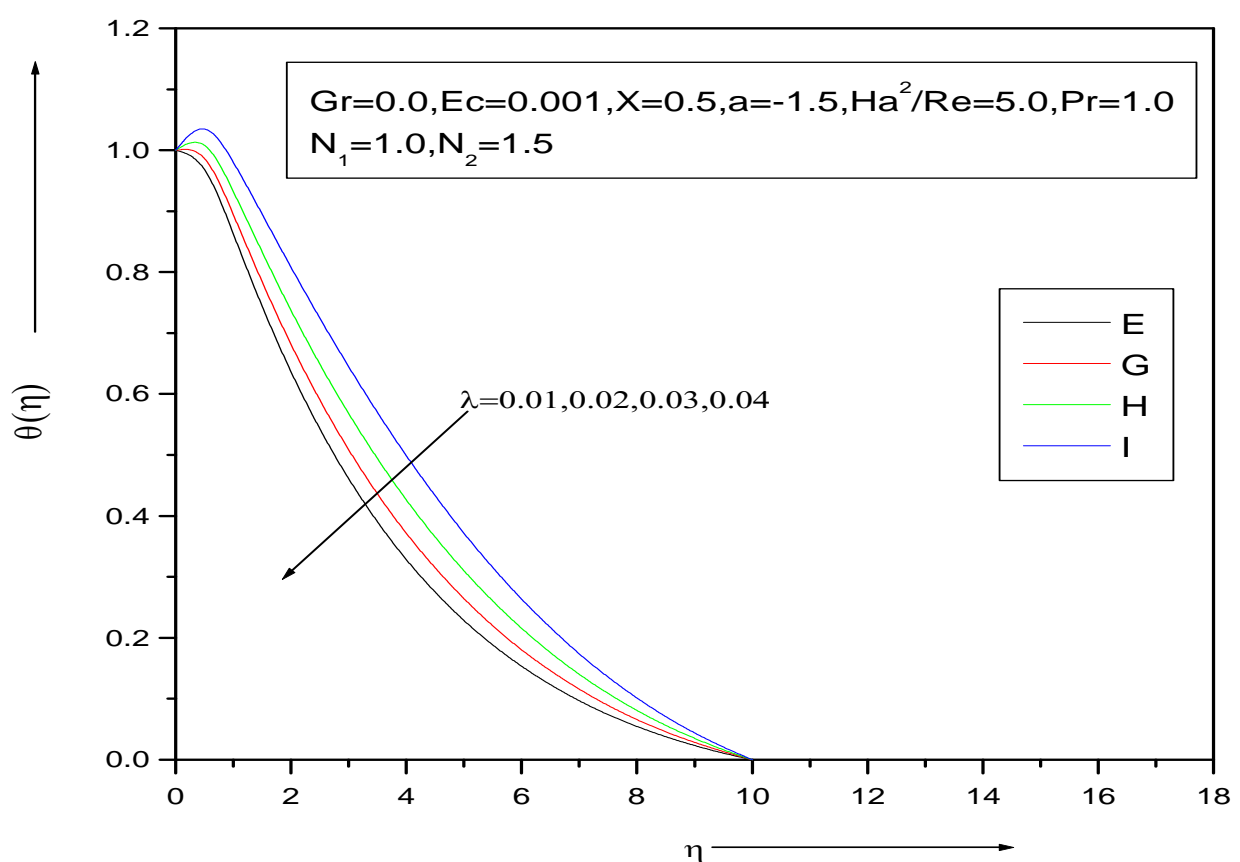

Fig13 Variation of temperature with $\eta$ for different values of $\lambda$ 


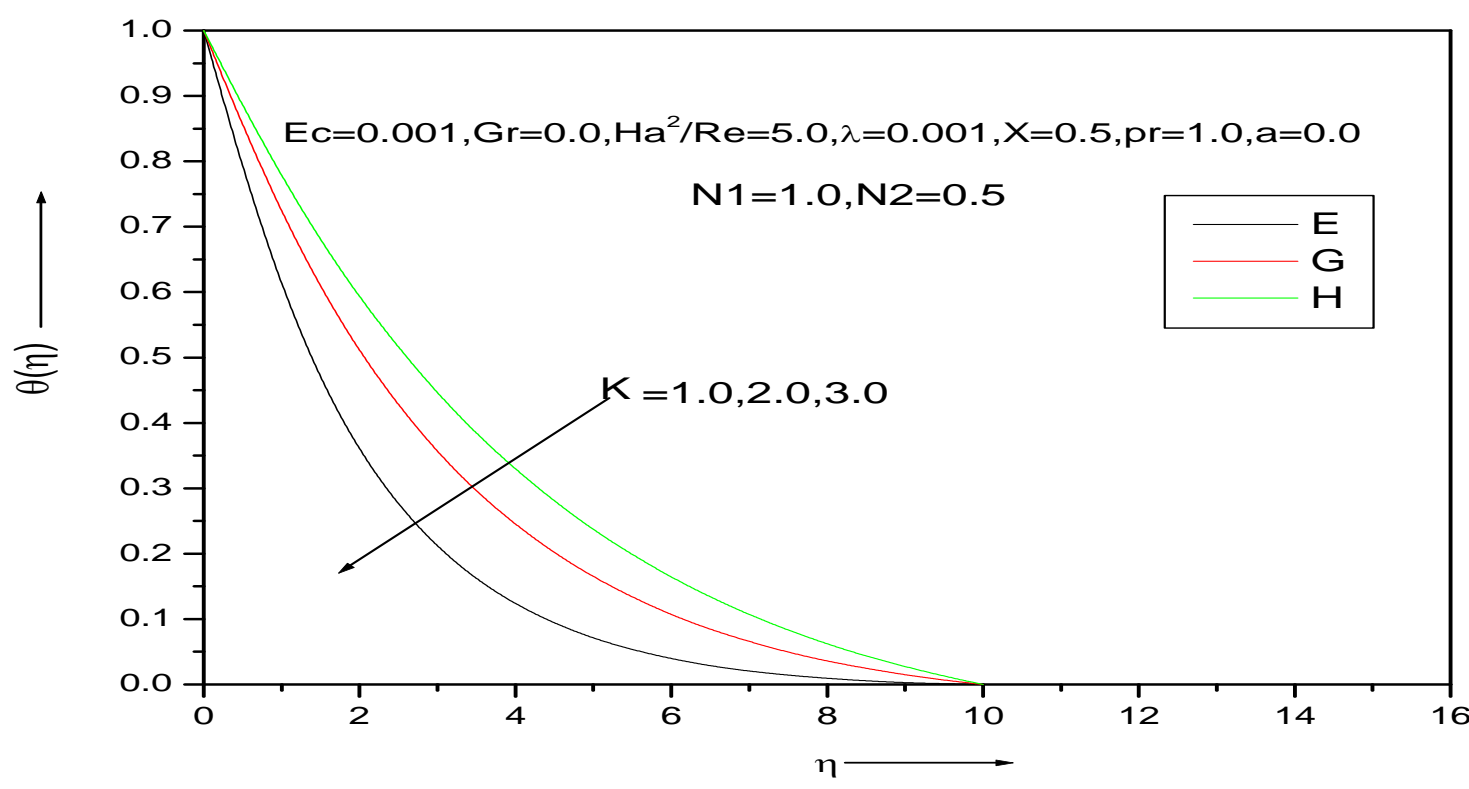

Fig14: Effects of K on the temperature profiles $\theta(\eta)$, where $\mathrm{K}=\frac{4 \sigma^{*} T_{\infty}^{3}}{k^{*} k}$ Radiation number

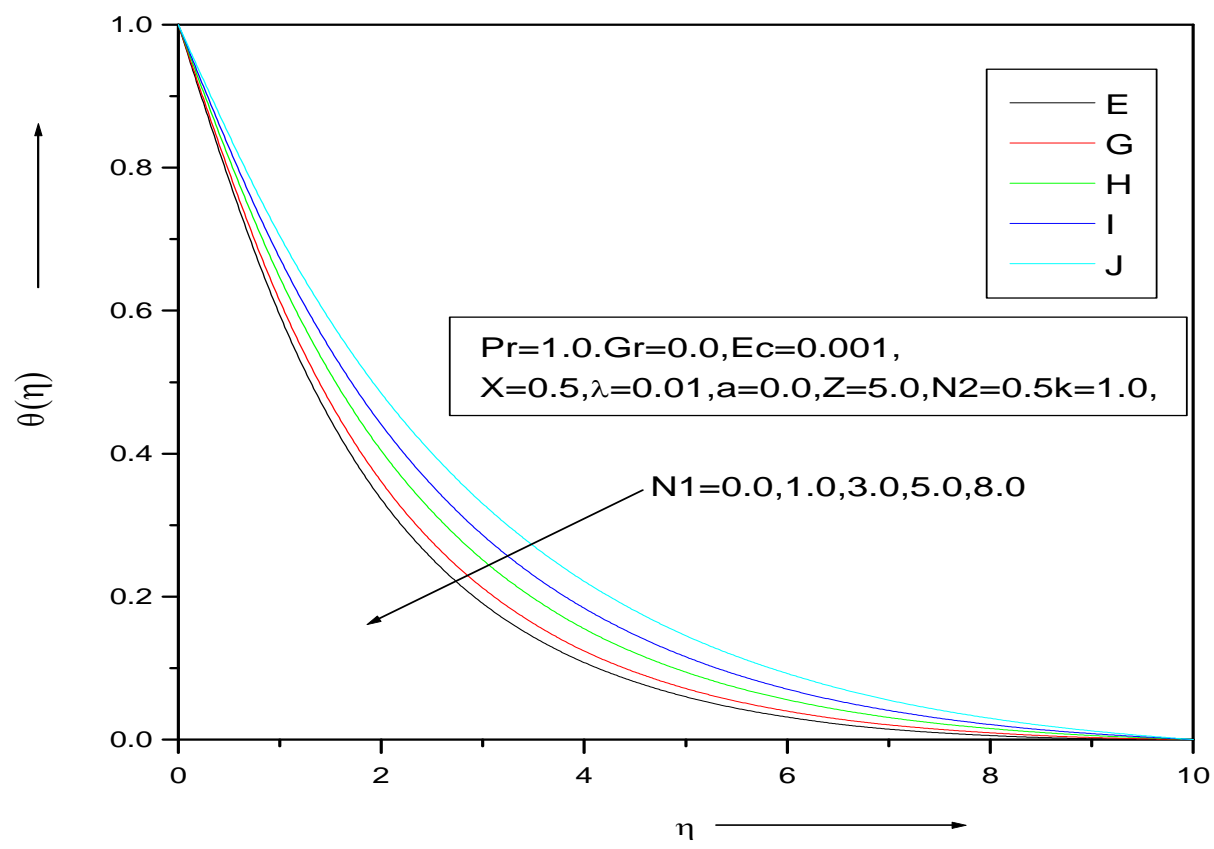

Fig 15: Effect of $\mathrm{N} 1$ on the temperature profiles $\theta(\eta)$ 


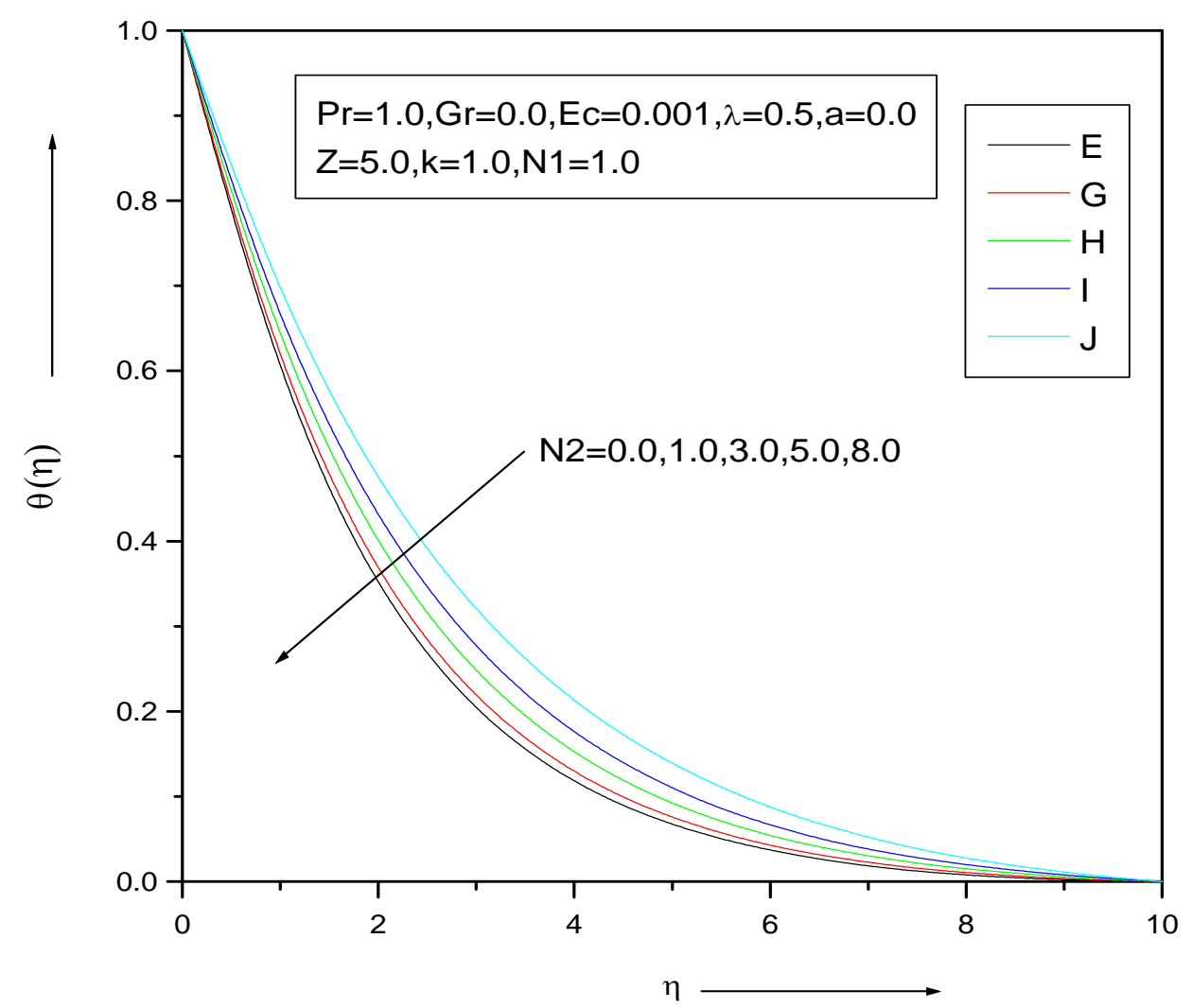

Fig 16: Effect of $\mathrm{N} 2$ on the temperature profiles $\theta(\eta)$

\section{CONCLUSIONS}

Due to the presence of porous parameter,the thermal boundary layer thickness increases.Effect of drag coefficient also enhance the thermal boundary layer thickness.

\section{REFERENCES}

[1]. Sakiadis.B.C, Boundary layer behaviour on continuous solid surface: I - Boundary layer equations for two dimensional and axisymmetric flows, AIChE. J. 7(1961)26-28. [2]. Sakiadis.B.C, Boundary layer behaviour on continuous solid surface: II - Boundary layer on a continuous flat surface AIChE.J.7 (1961) 221-225.

[3]. Kumaran.V, Ramanaiah.G, A note on the flow over a stretching sheet, Acta Mech. 116 (1996) 229-233.

[4]. Ali.M.E, On thermal boundary layer on a power law stretched surface with suction or injection Int. J. Heat Mass Flow 16 (1995) 280-290.
[5]. Elbashbeshy.E.M.A, Heat transfer over an exponentially stretching continuous surface with suction, Arch. Mech. 53 (6) (2001) 643-651.

[6]. Magyari. E, Keller .B, Heat and mass transfer in the boundary layers on an exponentially stretching continuous surface, J.Phys. D Appl. Phys.32 (1999) 577-585.

[7]. Khan.S.K, Sanjayanand.E, Viscoelastic boundary layer flow and heat transfer over an exponentially stretching sheet, Int. J. Heat Mass Transfer 48 (2005) 1534-1542.

[8]. Sanjayanand.E, Khan.S.K, On heat and mass transfer in a viscoelastic boundary layer flow over an exponentially stretching sheet, Int. J. Therm. Sci. 45 (2006) 819-828.

[9]. A.Raptis, G.Tzivanidis,N.Kafousias,Free convection and mass transfer flow through a porous medium bounded by an infinite vertical limiting surface with constant suction, Letter Heat mass transfer.8 (1981) 417-424.

[10]. A.Bejan,K.R.Khair,Heat and mass transfer by natural convection in porous medium,Int.J.Heat mass transfer 28 (1985) 909-918.

[11]. P.Forchheimer, Wasserbewegungdurch Boden, ForschHft.Ver Dt.Ing.45(1901) 1782-1788. 
[12]. O.A.Plumb, J.C.HUenefeld,Non-Darcy natural convection from heated surfaces in saturated porous media ,Int.J.Heat mass transfer 27 (1984)717-722.

[13]. D.A.S.Rees, I. Pop, A note on free convection along a vertical wavy surface in porous medium, ASME J.Heat transfer 116 (1994) 505-508.

[14]. D.A.S.Rees, I.Pop, Non-darcy natural convection from a vertical wavy surface in a porous medium, Transport porous medium 20 (1995) 133-141.

[15]. F.K.Tsou,E.M.Sparrow,R.J.Goldstien, Flow and heat transfer in the boundary layer on a continuous moving surface.Int.J.Heat mass transfer.10 (1967) 219-223.

[16]. P.S.Gupta,A.S.Gupta,Heat and mass transfer on stretching sheet with suction or blowing .Can.J.Chem.Eng.55 (1977) 744-746.

[17]. H.J.Andersson,K.H.Bech,Magnetohydrodynamic flow of a power-law fluid over a stretching sheet.Int.J.Nonlinear Mech.27 (1992) 929-936.

[18]. K.B.Pavlov,Magnetohydrodynamic flow of an incompressible viscous fluid caused by the deformation of a plane surface .Magan.Gidrodin.4 (1974) 146-147.

[19]. M.S.Abel,N.Mahesha,Heat transfer in MHD viscoelastic fluid flow over a stretching sheet with variable thermal conductivity,non-uniform heat source and radiation ,Appl.Math.Model.32 (2008) 1965-1983. 